\title{
Integumentary System Finding
}

National Cancer Institute

\section{Source}

National Cancer Institute. Integumentary System Finding. NCI Thesaurus. Code C36281.

Symptoms, physical examination results, and/or laboratory test results related to the integumentary system. 